\title{
Un modèle pluie-débit journalier à trois paramètres
}

\author{
Edijatno stagiaire \\ C. Michel IGREF \\ CEMAGREF - Antony
}

\section{Introduction}

Quoi de plus naturel que la pluie qui tombe, s'évapore, s'infiltre, ruisselle et contribue ainsi au flot qui parcourt les rivières?

Si l'on raconte à l'homme de la rue que la description de ce processus exige le recours à des équations complexes, pose des problèmes numériques difficiles aux mathématiciens, et absorbe l'énergie de nos ordinateurs modernes, sans aucun doute, il en sera très étonné.

Un peu de ce bon sens devrait éclairer notre approche de l'hydrologie et nous convaincre du caractère déplacé de la situation où nous sommes lorsque nous bâtissons ces énormes modèles, où nous cherchons à nous raccrocher toujours plus profondément aux fondements de la mécanique des fluides.
C'est dans cet état d'esprit que nous nous proposons de chercher un modèle simple et commode d'emploi, adapté à la simplicité du phénomène naturel. Le problème que nous cherchons à résoudre est de simuler les débits journaliers en un point d'une rivière à partir de la connaissance de la pluie journalière moyenne reçue par le bassin versant relatif au point considéré. Le modèle correspondant est dit global car on ne cherche pas à prendre en compte les irrégularités dans la répartition spatiale des pluies. Notre ambition est d'arriver à une simulation pas trop décevante en utilisant une représentation la plus simple possible du processus pluie-débit, dépendante d'un très faible nombre de paramètres, qu'il restera à caler pour s'adapter à la rivière que l'on veut étudier. Nous avons exclu du champ de notre recherche les bassins où la neige joue un rôle significatif.

\section{A daily rainfall-runoff model with three parameters}

The specialized literature may give the impression that the rainfall-runoff process is considered as a quite solved problem. However, when we examine the current models we notice that they are rather cumbersome, difficult to calibrate and not very satisfying by their results. Therefore, one may wonder whether it wouldn't be possible to get the same results with far simpler models. The present paper reports an attempt to conceive such a model. 


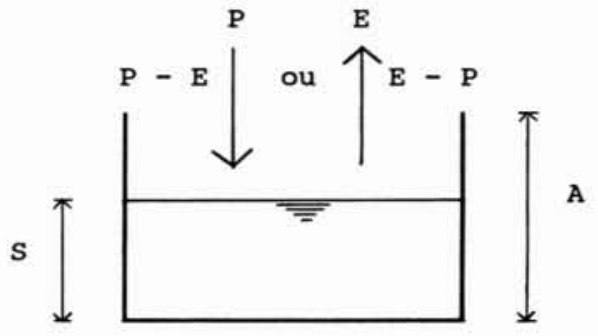

1. Le réservoir sol.

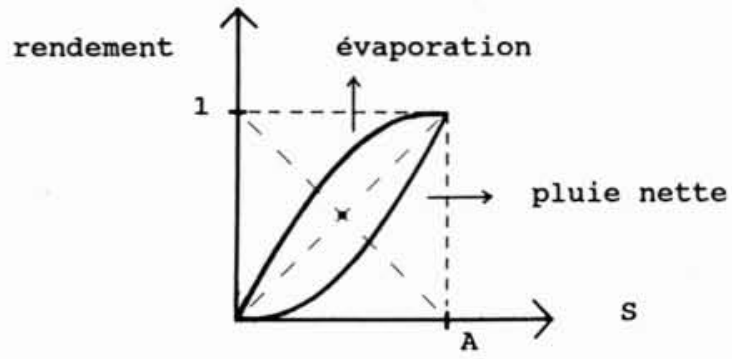

2. Rendements de la pluie et de l'évaporation.

\section{Un modèle à réservoirs}

Le modèle sur lequel a porté notre recherche, dénommé GR3 appartient à la classe générale des modèles à réservoirs et dérive notamment du modèle CREC mis au point par Y. CORMARY et A. GUILBOT en 1969.

Une version simplifiée du modèle GR3 a été élaborée pour rendre compte de la transformation pluie-débit sur le bassin de l'Orgeval (C. MiCHEL, 1983).

Le présent modèle a une portée plus générale puisqu'il vise à représenter le processus de production des débits pour des bassins versants allant de quelques $\mathrm{km}^{2}$ à quelques milliers de $\mathrm{km}^{2}$, tout en excluant, comme dit précédemment, les bassins où le phénomène neigeux joue un rôle significatif.

L'architecture du modèle repose sur deux réservoirs et un hydrogramme unitaire :

- le réservoir-sol ayant comme seule sortie le prélèvement occasionné par l'évaporation potentielle. Ce réservoir commande la répartition de la pluie nette entre luimême et le sous-modèle de routage ;

- un hydrogramme unitaire, décrivant la propagation des débits depuis leur formation à partir des pluies nettes jusqu'au deuxième réservoir qui est :

- le réservoir-eau-gravitaire. Ce réservoir reçoit les débits routés selon l'hydrogramme unitaire. Il a comme seule sortie le débit de la rivière et sa loi de vidange est du type quadratique.

Cet ensemble de trois opérateurs dont chacun dépend d'un seul paramètre semble être, à l'heure actuelle, le schéma le plus simple pour rendre compte de façon acceptable de la transformation pluie-débit. Les tentatives de «perfectionnement » de ce schéma, en vue de lui conférer une qualité de fonctionnement plus complexe, n'ont pas été jugées suffisamment pertinentes pour être retenues.

Nous allons présenter d'abord successivement les deux réservoirs du modèle GR3.

\section{Le réservoir-sol ( $f i g .1$ )}

Il est caractérisé par sa capacité $A$, premier paramètre du modèle GR3.

Son niveau $S$ est soumis soit à la pluie $(P-E)$ soit à l'évaporation potentielle $(E-P)$, selon que ces termes sont positifs. Dans ce qui suit $P$ et $E$ désigneront ces différences positives.

Compte tenu de l'extrême simplicité du modèle, l'évaporation potentielle est une simple moyenne interannuelle de l'évaporation calculée selon la formule de H.L. Penman. Des valeurs décadaires moyennes sur la période 1951-1980 peuvent être obtenues auprès du service de la Météorologie nationale.

Un court sous-programme permet d'en déduire des valeurs journalières par ajustement de courbes du second degré.

Il en résulte que les seules données datées dont on ait besoin pour faire fonctionner le modèle sont les pluies journalières éventuellement moyennées s'il existe plusieurs postes pluviométriques sur le bassin versant.

Le niveau $S$ du réservoir-sol permet de définir la fraction de pluie qui accédera au réservoir-eau-gravitaire. Cette fraction est définie en gros par le rapport :

$$
\left[\frac{S}{A}\right]^{2} \text {. }
$$

Le complément à 1 de cette fraction entre dans le réservoir-sol. Cette relation en valeurs instantanées peut être intégrée sur le pas de temps sans complication notoire. Cela permet de tenir compte de l'évolution de $S$ au cours du jour où il reçoit la pluie $P$. On obtient ainsi :

$$
\Delta S=A \tanh (P / A)\left[\frac{1-(S / A)^{2}}{1+(S / A) \tanh (P / A)}\right] .
$$

Le niveau du réservoir-sol permet également de définir l'évaporation réelle qu'il pourra délivrer. Cette évapora- 
tion réelle est une fraction de l'évaporation potentielle ayant des caractéristiques symétriques de la fraction représentée par (1):

$$
\frac{S}{A}\left[2-\frac{S}{A}\right] .
$$

On peut affiner cette relation en l'intégrant sur le pas de temps $\Delta t$ :

$$
\mathrm{d} S=-\frac{S}{A}\left[2-\frac{S}{A}\right] \mathrm{d} E
$$

qui donne approximativement :

$$
\Delta S=-E \frac{S}{E+A /(2-S / A)} .
$$

Les deux fractions concernant le rendement de la pluie et le rendement de l'évaporation illustrent la différence majeure entre les deux processus: le rendement des pluies n'approche l'unité que tardivement, lorsque $S$ est proche de $A$. Le rendement de l'évaporation réelle n'approche la valeur 0 que lorsque $S$ est proche de 0 . Ces deux courbes sont symétriques par rapport au point $(A / 2,1 / 2)$ (fig. 2).

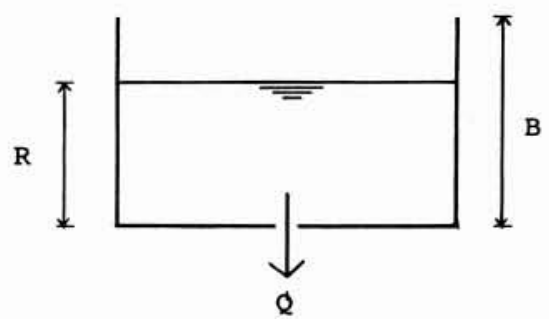

3. Le réservoir-eau-gravitaire.

\section{Le réservoir-eau-gravitaire (fig. 3)}

C'est un réservoir de type quadratique qui délivre le débit journalier de la rivière. Il est caractérisé par sa « rétention maximale à un jour » $B$, deuxième paramètre du modèle GR3.

Ce réservoir reçoit, en début de pas de temps, le débit délivré par l'hydrogramme unitaire. Le niveau $R$ du réservoir-eau-gravitaire détermine le débit $Q$ (lame d'eau journalière) qu'il peut relâcher :

$$
Q=\frac{R^{2}}{R+B} .
$$

Cette relation résulte de l'intégration sur le pas de temps $\Delta t$ d'une relation quadratique :

$$
-\frac{\mathrm{d} R}{\mathrm{~d} t}=k R^{2} \text {. }
$$

La loi de vidange du réservoir-eau-gravitaire reproduit assez bien les courbes de décrue telles que l'on peut les observer sur les hydrogrammes réels.

Cependant, ce schéma ne permet pas de simuler une montée de crue. C'est le problème dont nous allons décrire une solution possible par l'introduction en amont de ce réservoir d'un opérateur hydrogramme unitaire de type particulier, comme annoncé plus haut.

\section{Le temps d'accès au réservoir-eau-gravitaire}

Dans la version initiale du modèle GR3 ce temps d'accès était présent sous la forme d'un décalage entre l'apparition d'une pluie nette et le moment de son entrée dans le réservoir-eau-gravitaire (fig. 4).

On obtient ainsi un troisième paramètre égal à la durée du décalage entre l'apparition de la pluie brute $P$ et l'introduction de la pluie nette qui en résulte dans le réservoir $R$ pour produire, au même moment, le débit $Q$.

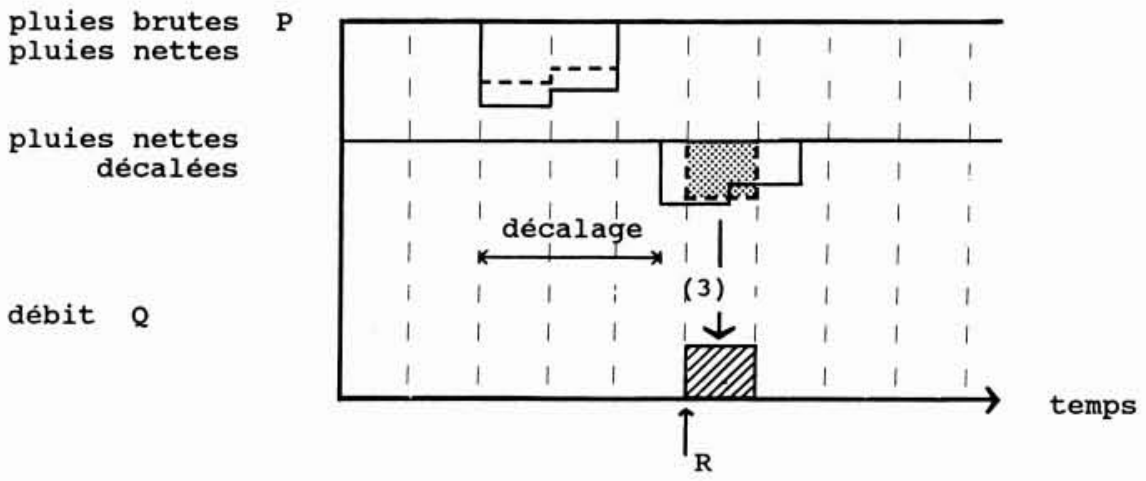

4. Décalage simple des pluies nettes. 


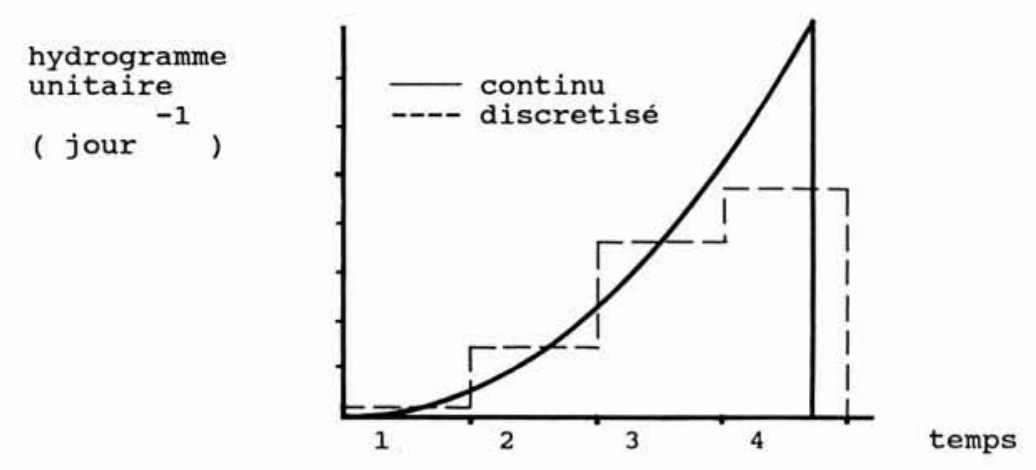

5. Hydrogramme unitaire simulant le transfert au réservoir eaugravitaire.

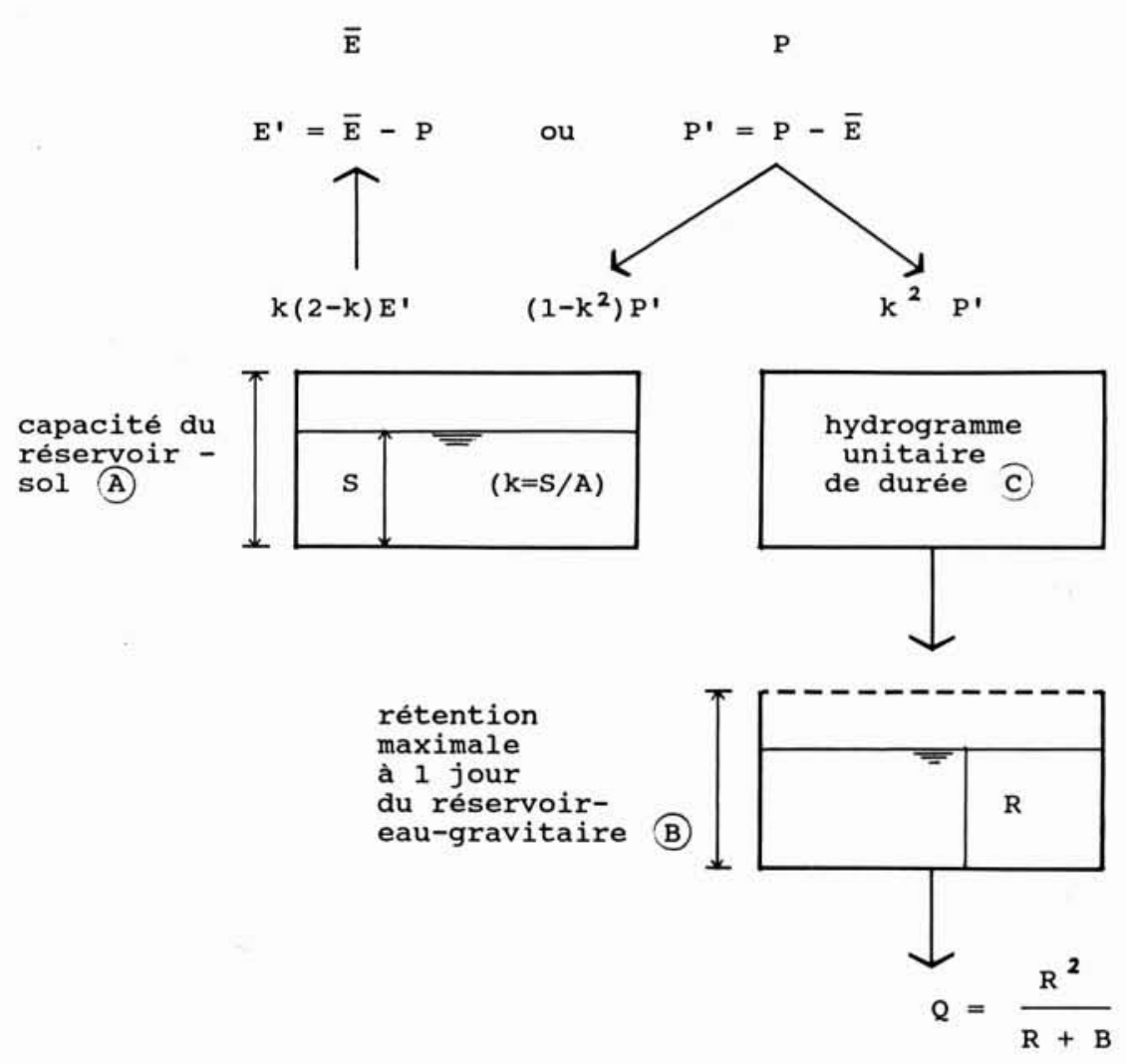

6. Schéma d'ensemble du modèle GR3. 
Cette solution est tout à fait utilisable mais un peu brutale dans son application puisqu'aucun effet de la pluie $P$ ne pourra apparaître avant le décalage prévu, et que par contre, cet effet sera total le jour suivant.

La solution proposée est de réaliser un décalage progressif par utilisation de l'opérateur classique qu'est l'hydrogramme unitaire.

Nous proposons un hydrogramme unitaire très simple, dépendant d'un seul paramètre qui est sa durée $C$.

C'est donc le troisième et dernier paramètre du modèle GR3, il est exprimé en jours.

Sous sa forme continue cet hydrogramme unitaire est de forme parabolique. Appelons $q(t)$ son ordonnée en fonction du temps $t$, il s'écrit alors :

$$
q(t)=\frac{3}{C^{3}} t^{2} .
$$

On doit en établir la version discrétisée pour son utilisation dans GR3 : si $j$ représente le temps en jour on a pour $j$ allant de 1 à $N$ (valeur entière de $C$ ) :

$$
q_{j}=\int_{j-1}^{j} q(t) \mathrm{d} t=\frac{3 j^{2}-3 j+1}{C^{3}}
$$

et enfin $($ si $N<C)$ :

$$
q_{N+1}=1-\left[\frac{N}{C}\right]^{3}
$$

La figure 5 représente l'hydrogramme unitaire proposé avec sa version discrétisée.

\section{Schéma d'ensemble du modèle GR3}

Les trois opérateurs du modèle, une fois rassemblés, conduisent au modèle GR3 tel qu'il est représenté sur la figure 6.

\section{Critère d'appréciation du modèle}

Le modèle tel qu'il vient d'être présenté permet donc de produire une série chronologique de débits $\hat{Q}_{j}$ à partir d'une série de pluies $P_{j}$. Le modèle sera d'autant plus satisfaisant que les débits $\hat{Q}_{j}$ ainsi calculés seront proches des débits $Q_{j}$ effectivement observés. Apprécier la validité du modèle consiste à juger de la proximité des deux séries chronologiques $\hat{Q}_{j}$ et $Q_{j}$. Ceci peut être fait visuellement mais on ne peut éviter de définir un critère numérique qui permettra de faire des comparaisons entre modèles et aussi de caler le modèle c'est-à-dire de trouver les valeurs les plus adéquates à affecter aux paramètres $A, B, C$.

Après une réflexion portant sur un assez grand nombre d'essais, il est apparu que le critère simple des moindres carrés est encore le plus intéressant :

$$
\text { Critère à minimiser : } \sum_{j=1}^{n}\left[Q_{j}-\hat{Q}_{j}\right]^{2}
$$

( $n$ étant la durée de la série chronologique).

Il est utile de disposer d'un critère adimensionnel facilitant la comparaison d'un bassin versant à l'autre.

Pour ce faire, calculons d'abord la moyenne quadratique des erreurs, $U$, telle que :

$$
n U^{2}=\sum_{j=1}^{n}\left[Q_{j}-\hat{Q}_{j}\right]^{2} .
$$

On peut calculer également la moyenne quadratique des pluies, que nous noterons $V$ :

$$
n V^{2}=\sum_{j=1}^{n} P_{j}^{2}
$$

Un critère adimensionnel commode est le nombre $T$ exprimé en pourcentage et donné par :

$$
\text { En principe, } \quad 0 \leqslant T \leqslant 100
$$

et le modèle est d'autant meilleur que $T$ est proche de 100. Sur les bassins versants où le modèle GR3 a été testé on obtient des valeurs de $T$ sur une période de calage de trois à cinq années variant de 80 à $96 \%$ environ. Ces résultats sont tout à fait honorables même lorsqu'on les compare aux performances de modèles beaucoup plus sophistiqués.

\section{Calage du modèle}

Le calage du modèle pose le problème de la durée de mise en route. Si l'on connait les conditions initiales, c'est-à-dire le débit initial $Q_{0}$, on ne peut en déduire que le niveau initial du réservoir-eau-gravitaire, en utilisant la relation (3) :

$$
R_{0}=\frac{Q_{0}}{2}\left[1+\sqrt{1+4 B / Q_{0}}\right] .
$$

Le niveau initial du réservoir-sol doit faire l'objet d'une supputation.

Selon les essais déjà réalisés on pourra estimer $S_{0}$ selon le mois du démarrage de la simulation, conformément au tableau ci-dessous.

\section{Initialisation du réservoir-sol}

\begin{tabular}{|ccccccccccccc|}
\hline Mois & 1 & 2 & 3 & 4 & 5 & 6 & 7 & 8 & 9 & 10 & 11 & 12 \\
\hline So/A & .80 & .85 & .84 & .74 & .62 & .44 & .28 & .16 & .19 & .38 & .50 & .67 \\
\hline
\end{tabular}




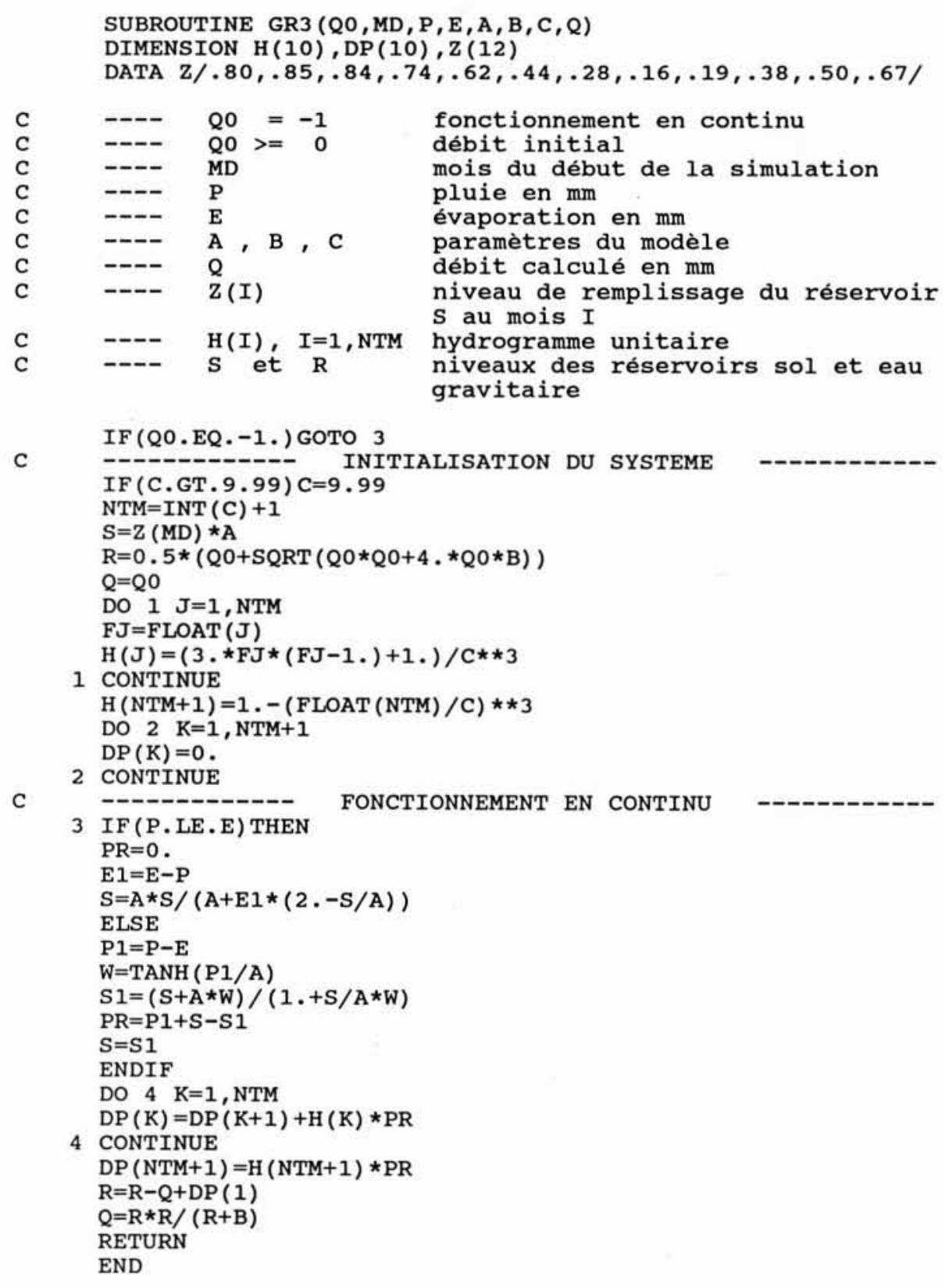

7. Liste du sous-programme GR3 (Fortran 77). 
Ces indications restent très grossières et il s'ensuit qu'une erreur non négligeable peut être commise en faisant ce choix arbitraire. Cependant, au fur et à mesure que le modèle fonctionne, il s'effectue un réajustement progressif du niveau $S$ du réservoir-sol. Ainsi, une mauvaise initialisation du réservoir-sol voit ses effets s'atténuer au cours du temps. Deux solutions peuvent être adoptées pour tenir compte d'une mauvaise initialisation éventuelle :

a) considérer que le niveau initial du réservoir-sol est un paramètre à caler ;

b) ne pas tenir compte du début de la simulation, soumise à l'influence de l'initialisation, et dénommée période de mise en route. C'est cette deuxième solution qui a été choisie en adoptant une période de mise en route d'une année (il faut traverser une saison de hauts débits et une saison d'étiage, pour espérer une remise à niveau correcte du réservoir-sol).

Un algorithme très simple a été développé spécialement pour le calage de GR3. Il s'apparente aux techniques de gradient et fonctionne sur les logarithmes des paramètres $A, B, C$ à caler. Une telle transformation est souvent nécessaire pour rendre plus efficace la recherche de l'optimum.

\section{Contrôle du modèle}

En général, il est essentiel de distinguer parmi les données disponibles, deux périodes d'égale importance, dont l'une servira au calage du modèle et l'autre au contrôle sur des données étrangères au calage.

Très souvent, le critère de jugement du modèle se détériore très sensiblement lorsque l'on passe de la période de calage à la période de contrôle. Et cela d'autant plus que le modèle est sophistiqué et contient un grand nombre de paramètres ajustables à une situation donnée : c'est le phénomène de "surcalage».

A l'expérience, il est apparu que le modèle GR3 ne conduisait pas à une remise en cause du modèle sur la période de contrôle et cela s'explique probablement par la grande rusticité du modèle et son faible nombre de paramètres. Cela permet de fonctionner de façon fiable, même avec une durée de données disponibles ne dépassant guère trois années (dont une pour la mise en route).

\section{Présentation informatique du modèle GR3}

Les développements précédents permettent d'avoir une idée assez précise du modèle GR3. Cependant il est utile d'en avoir une description informatique, levant toute ambiguïté éventuelle. La figure 7 donne le sous-programme GR3 écrit en FORTRAN 77.

Les trois paramètres $A, B, C$ sont initialisés dans le programme principal. En l'absence de formules d'estimation a priori, on prend comme valeurs initiales pour le calage respectivement $150(\mathrm{~mm}), 50(\mathrm{~mm})$ et 1,5 (jour). La variable $Q_{0}$ permet de distinguer deux fonctionnements du sous-programme :

a) $Q_{0}>0$.

Ce cas correspond au début de la simulation d'une chronique continue de débits avec un jeu de paramètres fixé. $Q_{0}$ représente alors le débit initial. Le sous-programme prévoit l'initialisation du système, à savoir les niveaux des réservoirs sol et eau-gravitaire ainsi que les premières pluies nettes, rangées dans le vecteur $D P$.

b) $Q_{0}=-1$.

Ce cas correspond à la production continue des débits journaliers. On entre la pluie brute $P$ et l'évaporation potentielle $E$ et le sous-programme renvoie la valeur de $Q$, le débit calculé à la même date que $P$.

\section{Application au bassin de la Seine}

Une application au bassin de la Seine à Bar-sur-Seine (2 $340 \mathrm{~km}^{2}$, figure 8 ) est présentée ci-après.

8. Plan de situation du bassin de la Seine à Bar-sur-Seine.

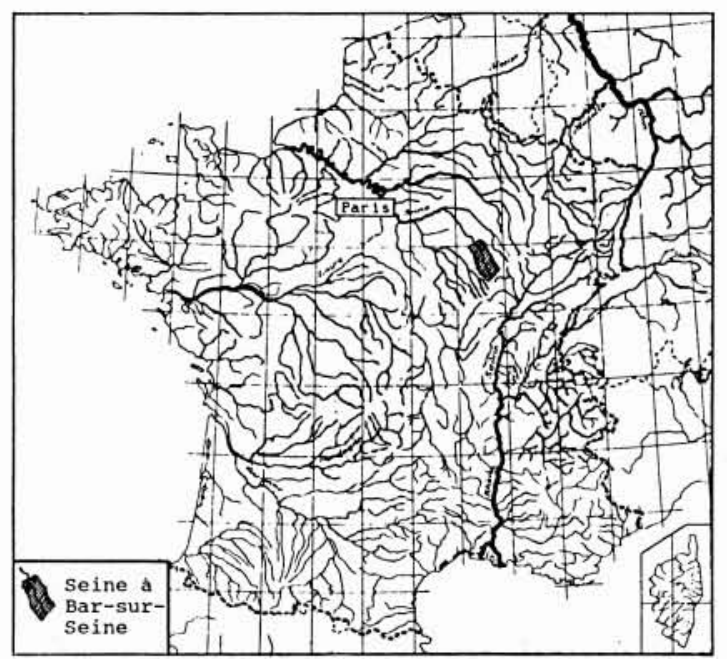


BAR SUR SEINE
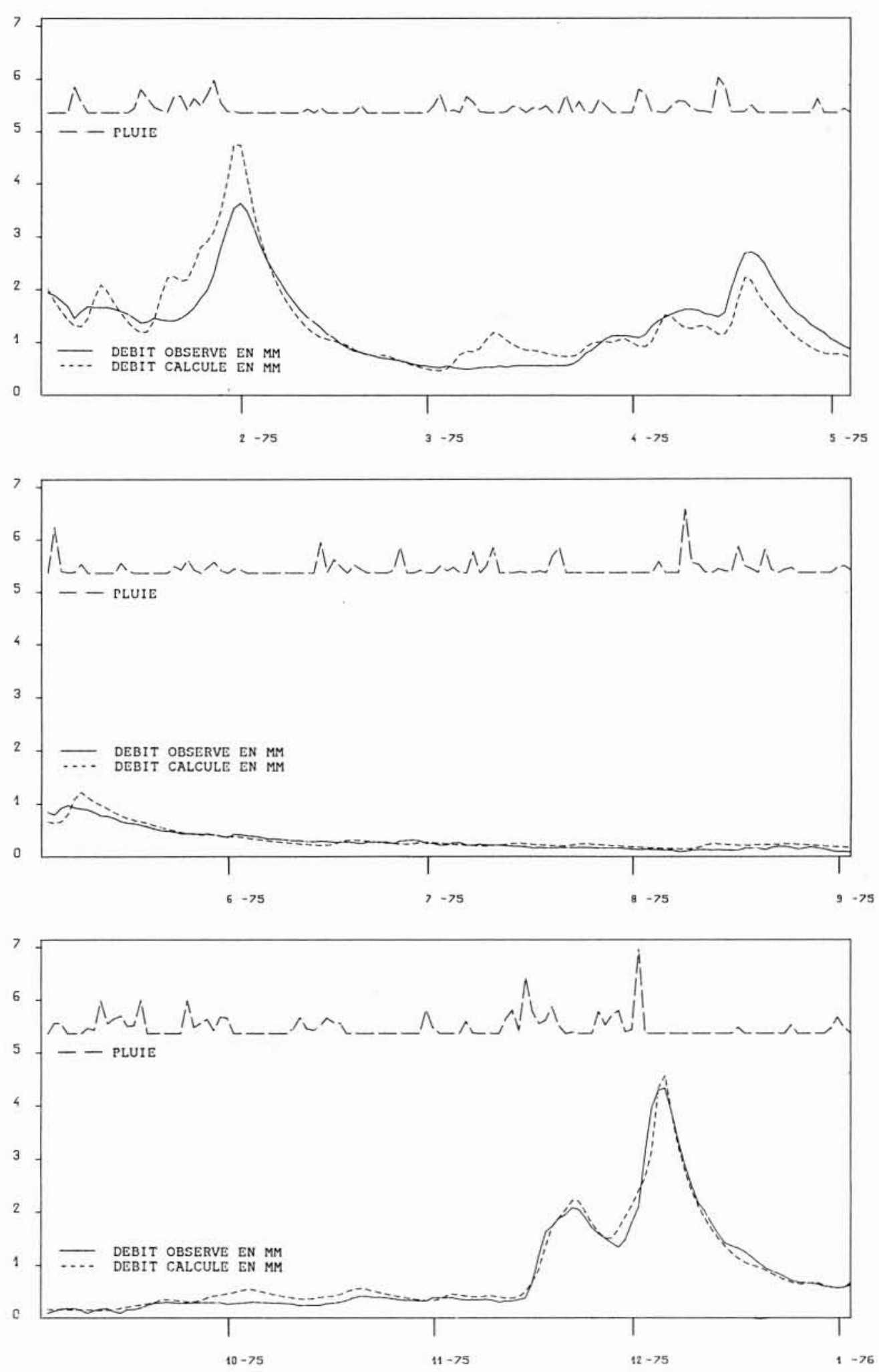

9. Simulation de l'année 1975 (trait pointillé). 
Un calage sur les années 1972 à 1976 a conduit aux valeurs suivantes des paramètres :

$$
A=340 \mathrm{~mm} ; B=530 \mathrm{~mm} ; C=3,3 \text { jours . }
$$

Le fonctionnement du modèle sur l'année 1975 est représenté sur la figure 9.

\section{Conclusion}

Concevoir un modèle pluie-débit journalier ne dépendant que de trois paramètres pouvait relever de la gageure. En fait le modèle GR3 prouve que cela est possible et conduit même à des résultats tout à fait présentables. Les avantages de la faisabilité d'un tel modèle sont évidents :

- commodité d'emploi

- faibles incertitudes à l'initialisation

- caractérisation d'un bassin par seulement trois « coordonnées"

- facilité de calage.

Un vaste domaine est alors ouvert à la recherche :

- explication des paramètres en fonction de caractéristiques identifiables du bassin versant ;

- étude de sensibilité des paramètres et identification de l'impact des activités humaines sur le cycle hydrologique ;
- simulation de longues séries de débits à partir de chroniques de pluies enregistrées et estimation des crues rares ;

- outil de comparaison pour des modèles plus sophistiqués.

\section{Remerciements}

Le Ministère de l'Environnement (SRETIE) a bien voulu accorder une subvention pour la recherche sur le modèle GR3 sur proposition de Monsieur Pierre-Alain RoCHE (thèse de Monsieur Edijatno).

\section{Edijatno,}

thésard de l'ENITRTS et de l'ULP de Strasbourg, en stage au CEMAGREF

Claude Michel,

IGREF à la Section hydrologie du CEMAGREF.

BP 121, 92164 Antony Cedex

Tél. : 40966121

\section{Références}

Y. Cormary, A. Guilbot, 1973 : Etude des relations pluiedébit sur trois bassins versants représentatifs, AISH publication $n^{\circ} 108$, Colloque de Madrid, juin 1973, pp. 265-279.

C. MiCHEL, 1983 : "Que peut-on faire en hydrologie avec un modèle à un paramètre? ", La Houille Blanche, $\mathrm{n}^{\circ} 1$, 1983, pp. 39-44. 


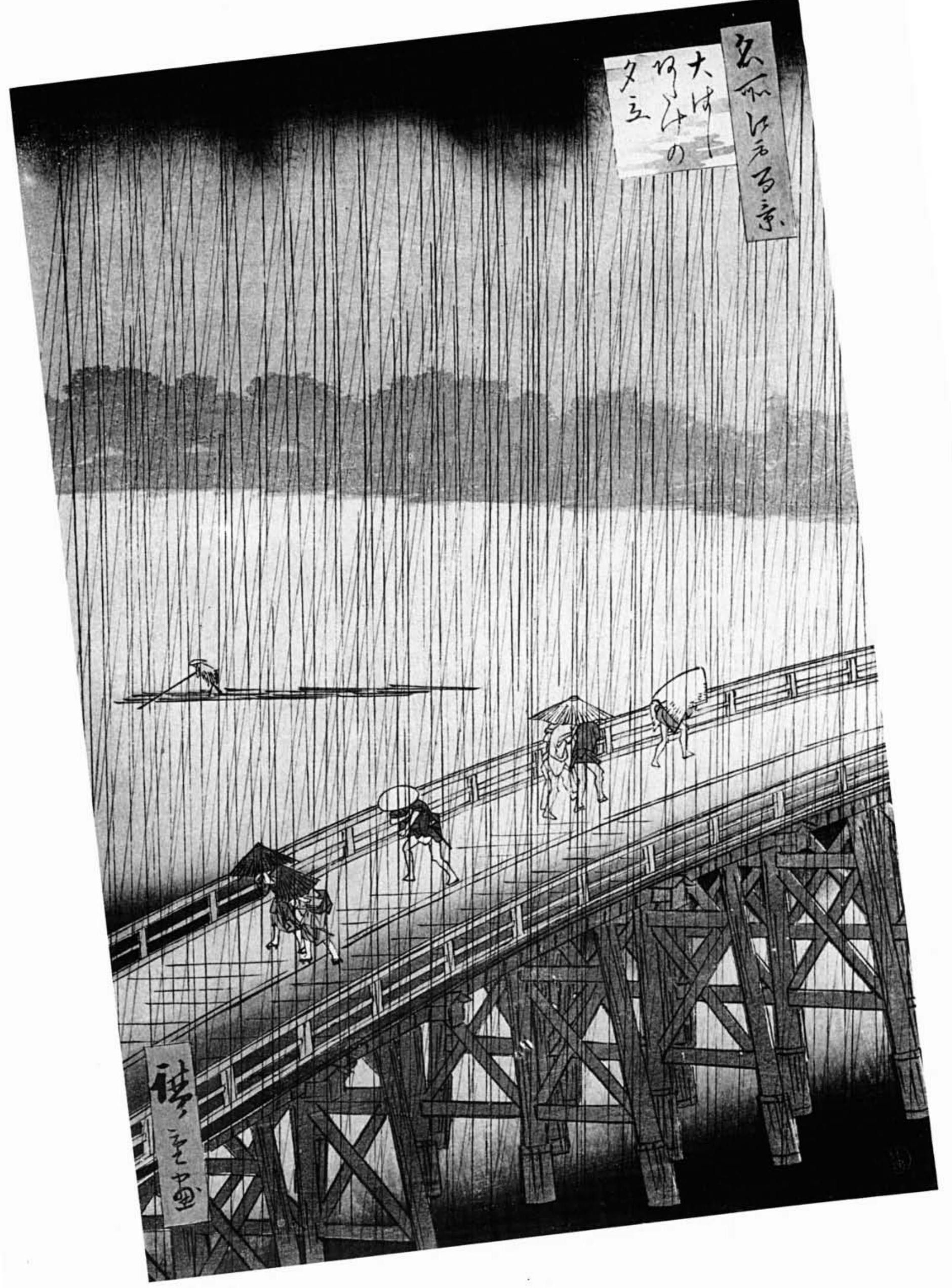

